ALEA, Lat. Am. J. Probab. Math. Stat. 14, 33-44 (2017)

DOI: 10.30757/ALEA.v14-03

\title{
Detecting a Path of Correlations in a Network
}

\author{
Ery Arias-Castro, Gábor Lugosi and Nicolas Verzelen
}

Department of Mathematics, University of California, San Diego, La Jolla, CA 92093-0112, United States

E-mail address: eariasca@ucsd.edu

URL: http://math.ucsd.edu/ eariasca/

ICREA and Department of Economics and Business, Pompeu Fabra University, 08010 Barcelona, Spain

E-mail address: gabor.lugosi@gmail.com

URL: http://www.econ.upf .edu/ Iugosi/

INRA, UMR 729 MISTEA,

34060 Montpellier, France

E-mail address: nicolas.verzelen@supagro.inra.fr

$U R L:$ http://nicolas.verzelen.free.fr/

\begin{abstract}
We consider the problem of detecting an anomaly in the form of a path of correlations hidden in white noise. We provide a minimax lower bound and a test that, under mild assumptions, is able to achieve the lower bound up to a multiplicative constant.
\end{abstract}

\section{Introduction}

Anomaly detection arises in many applications, including surveillance, the detection of suspicious objects from satellite images or sensor networks, as well as in medical imaging (e.g., tumor detection). While in some applications the object can be assumed to present a larger signal amplitude (e.g., pixel level in images), in other settings it manifests instead as correlations. For example, the object to be detected in an image has different texture but same average pixel amplitude; or in the case of the evolution of the price of a stock, an event could trigger more volatility instead of a change in the value of the stock. We call the problem of detecting the presence of a subset of observations with different mean from the rest

Received by the editors November 3, 2015; accepted November 14, 2016.

2010 Mathematics Subject Classification. 62C20, 94A13.

Key words and phrases. Detection of Anomalies, Graphs, Networks, Anomalous Correlations, Minimax Theory.

EAC was partially supported by the US National Science Foundation (DMS 1223137, 1120888). GL was partially supported by the Spanish Ministry of Economy and Competitiveness, Grant MTM2015-67304-P and FEDER, EU. NV was partially supported by the French Agence Nationale de la Recherche (ANR 2011 BS01 01001 projet Calibration). 
the detection-of-means problem. We call the problem of detecting the presence of a subset of unusually correlated observations the detection-of-correlations problem.

The detection-of-means problem has been extensively studied in the literature, both applied and theoretical. Papers that develop theory include Arias-Castro et al. (2008); Addario-Berry et al. (2010); Arias-Castro et al. (2011, 2005); Walther (2010); Desolneux et al. (2003). The detection-of-correlations problem has drawn less attention, and while the applied literature is sizable, few papers develop theory beyond the one-dimensional case of change-point detection in times series. In a few recent papers, we developed elements of the first minimax theory, see AriasCastro et al. (2012, 2015a,b). In the present paper we focus on detecting a path of correlations in a general graph. This setting could model an attack in a computer network, see Mukherjee et al. (1994); Zhang and Lee (2000). The corresponding detection-of-means setting was considered in Arias-Castro et al. (2008).

The remainder is organized as follows. In Section 2, after formalizing the problem, we derive a lower bound when the correlation parameter is known and then propose a testing procedure which achieves this lower bound (up to a multiplicative constant depending on some graph characteristics) without knowledge of the correlation parameter. In Section 3 we specialize our general results to the case of detecting a path of correlations in an integer lattice. The proofs are gathered in Section 4 .

\section{Setting and general results}

2.1. Formulation of the problem. We are given a graph $\mathcal{G}=(\mathcal{V}, \mathcal{E})$, with $\mathcal{V}$ denoting the set of nodes (or vertices) and $\mathcal{E} \subset \mathcal{V} \times \mathcal{V}$ denoting the set of edges. When finite, let $n=|\mathcal{V}|$ denote the number of nodes. We are also given a class of open, selfavoiding paths of $\mathcal{G}$, denoted $\mathcal{C}$. Recall that an open self-avoiding path in $\mathcal{G}$ is a sequence of nodes $S=\left(s_{1}, \ldots, s_{k}\right) \in \mathcal{V}^{k}$ such that $\left(s_{j}, s_{j+1}\right) \in \mathcal{E}$ for all $j$ and $s_{j} \neq s_{j^{\prime}}$ for all $j \neq j^{\prime}$. We observe a vector of random variables indexed by $\mathcal{V}$, denoted $X=\left(X_{i}\right)_{i \in \mathcal{V}}$, assumed to be standard normal. Under the null hypothesis all components of $X$ are independent. Under the alternative hypothesis, one of the paths $S \in \mathcal{C}$ is "anomalous", in which case $\left(X_{i}\right)_{i \in S}$ is an autoregressive model of order 1 with correlation coefficient $\psi \in(-1,1)$, while the other components of $X$ are still independent and $\left(X_{i}\right)_{i \in S}$ and $\left(X_{i}\right)_{i \notin S}$ are also independent. This means that, if $S=\left(s_{1}, \ldots, s_{k}\right)$, then $X_{s_{j+1}}-\psi X_{s_{j}}, j=1, \ldots, k-1$, are independent centered normal random variables with variance $1-\psi^{2}$. We denote the distribution of $X$ under $\mathcal{H}_{0}$ by $\mathbb{P}_{0}$. We denote the distribution of $X$ under $\mathcal{H}_{1}$ by $\mathbb{P}_{S, \psi}$ when $S \in \mathcal{C}$ is the anomalous set and $\psi$ is the autocorrelation coefficient.

A test is a measurable function $f: \mathbb{R}^{\mathcal{V}} \rightarrow\{0,1\}$. When $f(X)=0$, the test accepts the null hypothesis and it rejects it otherwise. The probability of type $I$ error of a test $f$ is $\mathbb{P}_{0}\{f(X)=1\}$. Under the alternative $(S, \psi) \in \mathcal{C} \times(-1,1)$, the probability of type II error is $\mathbb{P}_{S, \psi}\{f(X)=0\}$. In this paper we evaluate tests based on their worst-case risks. When the correlation coefficient $\psi$ is known, the risk of a test $f$ corresponding to the class $\mathcal{C}$ is defined as

$$
R_{\mathcal{C}, \psi}(f)=\mathbb{P}_{0}\{f(X)=1\}+\max _{S \in \mathcal{C}} \mathbb{P}_{S, \psi}\{f(X)=0\} .
$$

In this case, the minimax risk is defined as

$$
R_{\mathcal{C}, \psi}^{*}=\inf _{f} R_{\mathcal{C}, \psi}(f)
$$


where the infimum is over all tests $f$. When $\psi$ is only known to belong to an interval $\mathfrak{I} \subset(-1,1)$, it is more meaningful to define the risk of a test $f$ as

$$
R_{\mathcal{C}, \mathfrak{I}}(f)=\mathbb{P}_{0}\{f(X)=1\}+\max _{\psi \in \mathfrak{I}} \max _{S \in \mathcal{C}} \mathbb{P}_{S, \psi}\{f(X)=0\} .
$$

The corresponding minimax risk is defined as

$$
R_{\mathcal{C}, \mathfrak{I}}^{*}=\inf _{f} R_{\mathcal{C}, \mathfrak{I}}(f) .
$$

When $\psi$ is known (resp. unknown), we say that a test $f$ asymptotically separates the two hypotheses if $R_{\mathcal{C}, \psi}(f) \rightarrow 0$ (resp. $R_{\mathcal{C}, \mathfrak{I}}(f) \rightarrow 0$ ), and we say that the hypotheses merge asymptotically if $R_{\mathcal{C}, \psi}^{*} \rightarrow 1$ (resp. $R_{\mathcal{C}, \mathfrak{I}}^{*} \rightarrow 1$ ), as $n=|\mathcal{V}| \rightarrow \infty$. We note that, as long as $\psi \in \mathfrak{I}, R_{\mathcal{C}, \psi}^{*} \leq R_{\mathcal{C}, \mathfrak{I}}^{*}$ and that $R_{\mathcal{C}, \mathfrak{I}}^{*} \leq 1$, since the test $f \equiv 1$, that always rejects $\mathcal{H}_{0}$, has risk equal to 1.

In this paper, we characterize the minimax testing risk in the setting where $\psi$ is known $\left(R_{\mathcal{C}, \psi}^{*}\right)$ and in the setting when it is unknown $\left(R_{\mathcal{C}, \mathfrak{I}}^{*}\right)$. That is, we give conditions on $\mathcal{C}$ and $\mathfrak{I}$ under which the hypotheses merge asymptotically so that the detection problem is nearly impossible. We then exhibit a (nonstandard) test that asymptotically separates the hypotheses under essentially the same conditions.

2.2. A general lower bound. The main difference between the case of anomalous paths treated here and the case of anomalous blobs studied in Arias-Castro et al. (2015b) is that a lower bound for the latter can be developed based on a subclass of disjoint subsets. Here, however, a reduction to a subclass of disjoint paths is typically too severe. We thus develop a new lower bound tailored to the present situation, which, in particular, allows for possible anomalous subsets to intersect. For any prior distribution $\nu$ on $\mathcal{C}$, the minimax risk is at least as large as the $\nu$-average risk, $R_{\mathcal{C}, \psi}^{*} \geq \bar{R}_{\nu, \psi}^{*}$, where

$$
\bar{R}_{\nu, \psi}(f)=\mathbb{P}_{0}\{f(X)=1\}+\sum_{S \in \mathcal{C}} \nu(S) \mathbb{P}_{S, \psi}\{f(X)=0\} \quad \text { and } \quad \bar{R}_{\nu, \psi}^{*}=\inf _{f} \bar{R}_{\nu, \pi}(f) .
$$

The following result provides a lower bound on the latter.

Theorem 2.1. Let $\mathcal{C}$ be a class of open self-avoiding paths of $\mathcal{G}$ and let $\nu$ denote some prior over $\mathcal{C}$. Assume that $|\psi|<1 / 9$. Then

$$
\bar{R}_{\nu, \psi}^{*} \geq 1-\frac{1}{2} \sqrt{\mathbb{E}_{\nu \otimes \nu}[\exp (\lambda(\psi)|S \cap T|)]-1},
$$

where

$$
\lambda(\psi):=\frac{1}{4}\left[\left(\frac{1-|\psi|}{1-9|\psi|}\right)^{1 / 2}-\frac{1+|\psi|}{1-|\psi|}\right]
$$

and the expectation is with respect to $S, T$ drawn i.i.d. from $\nu$.

The function $\lambda$ is even and increasing on $(0,1 / 9)$, and $\lambda(1 / 10)=4 / 9$. Hence, the bound implies that

$$
\bar{R}_{\nu, \psi}^{*} \geq 1-\frac{1}{2} \sqrt{\mathbb{E}_{\nu \otimes \nu}\left[\exp \left(\frac{4}{9}|S \cap T|\right)\right]-1}, \quad \text { when }|\psi| \leq 1 / 10 .
$$

Remark 2.2. The condition on $\psi$ is likely to be an artifact of our proof technique. As is standard in the literature, the proof relies on bounding the variance of the likelihood ratio resulting from averaging the alternatives according to $\nu$. A more refined approach such as that of bounding the second moment of the likelihood 
ratio after truncation - a well-known technique in the detection-of-means setting first proposed by Yuri Ingster - might lead to a sharpening of this result, but the computations for the present case are daunting.

2.3. A general upper bound. A natural approach in related testing problems is the generalized likelihood ratio test. When $\psi$ is known, this test is based on rejecting the null hypothesis for large values of

$$
\max _{S \in \mathcal{C}} X_{S}^{\top}\left(\mathbf{I}_{S}-\boldsymbol{\Gamma}_{S}^{-1}(\psi)\right) X_{S}
$$

where $\boldsymbol{\Gamma}_{S}(\psi)$ denotes the covariance matrix of an autoregressive model of order 1 indexed by $S$ and with parameter $\psi$ and $X_{S}=\sum_{i \in S} X_{i}$. Establishing a useful performance bound for this test appears surprisingly challenging due to our lack of understanding of concentration properties of the test statistic under the null hypothesis. In particular, our effort to combine the union bound with a standard concentration bound for Gaussian quadratic forms (i.e., Gaussian chaoses of order 2 ) were inconclusive. The situation is even more complicated when $\psi$ is unknown.

However, we were able to craft and analyze an ad-hoc test based on pairwise comparisons of consecutive values along a path. For simplicity assume that all paths in $\mathcal{C}$ are of same length $k$. (When this is not the case, typically the test needs to be repeated for all possible lengths and the resulting multiple testing situation is resolved by applying Bonferroni's method.) Fix a threshold $t>0$. For $S=\left(s_{1}, \ldots, s_{k}\right) \in \mathcal{C}$, define

$$
V_{t, S}=\sum_{j=1}^{k-1} V_{t, S}(j), \quad V_{t, S}(j)=\mathbb{I}\left\{\left|X_{s_{j+1}}-X_{s_{j}}\right| \leq \sqrt{2} t\right\}
$$

and consider the statistic and corresponding test

$$
V_{t}^{*}=\max _{S \in \mathcal{C}} V_{t, S}, \quad f_{t}=\mathbb{I}\left\{V_{t}^{*}>k / 2\right\} .
$$

For $t \geq 0$, define $p_{t}=2 \mathrm{~N}(t)-1$, where $\mathrm{N}$ denotes the standard normal distribution function. Also, define the function $h(x)=x-\log (x)-1$.

Proposition 2.3. There is a sequence of reals $\left(r_{k}\right)$, with $r_{k} \rightarrow 0$ as $k \rightarrow \infty$, such that the following is true. Consider any setting where $\mathcal{C}$ is a class of (self-avoiding) paths of length $k$. Let $t=t(k)>0$ be largest such that $h\left(2 p_{t}\right) \geq \frac{8}{k} \log (|\mathcal{C}|) \vee 1$. If $\psi \geq 1-\left(t / \mathrm{N}^{-1}(4 / 5)\right)^{2}$, the test $f_{t}$ defined in (2.2) satisfies $R_{\mathcal{C}, \psi}\left(f_{t}\right) \leq r_{k}$.

Remark 2.4. The proposition only applies to positive $\psi$ and in fact the test (2.2) is only useful in that case. To handle the case where $\psi$ is negative, we use instead the variant where $V_{t, S}(j)$ is replaced with $\mathbb{I}\left\{\left|X_{s_{j+1}}+X_{s_{j}}\right| \leq \sqrt{2} t\right\}$. The resulting test achieves a similar performance. In practice, if the sign of $\psi$ is a priori unknown, one can simply combine these two tests using a Bonferroni correction. In the rest of the paper we only consider $\psi>0$.

Remark 2.5. Computing the test statistic $V_{t}^{*}$ of $(2.2)$ is difficult, even when the starting point is given. Indeed, this problem is known as the prize collecting salesman problem or bank robber problem or reward-budget problem. Even in the case where the underlying graph is the integer lattice there are no known polynomialtime algorithms that solve it, although polynomial approximations do exist, see DasGupta et al. (2006). An alternative is the test based on the length of the 
longest path of significant adjacent correlations. This is inspired from some proposals in the detection-of-means setting of Arias-Castro and Grimmett (2013) and Arias-Castro et al. (2006). Quick calculations suggest that the test achieves a comparable theoretical performance.

\section{Special case: the lattice}

Consider the integer lattice

$$
\mathcal{V}=\{1, \ldots, m\}^{d}
$$

in dimension $d \geq 3$. Note that $n=m^{d}$ in this case. The story is a little different when $d=2$, and we refer the reader to the treatment in Arias-Castro et al. (2008) carried out in the detection-of-means setting, as we expect a similar phenomenon to hold in the present context. For simplicity, to guarantee that all nodes play a symmetric role, we take the lattice to be a torus.

3.1. Known starting point. Let $\mathcal{C}$ be the class of all self-avoiding paths with $k$ nodes in $\mathcal{V}$ starting at some given $v_{0} \in \mathcal{V}$. In that case, when $d \geq 3$, there is a constant $C>0$ such that, when $|\psi| \leq C$, the risk is at least $1 / 2$. To see this, let $\nu$ be a prior on $\mathcal{C}$ that has exponential intersection tails, which means that there exist some constants $\eta \in(0,1)$ and $C_{0}>0$ such that

$$
\mathbb{P}_{\nu \otimes \nu}(|S \cap T| \geq \ell) \leq C_{0} \eta^{\ell}, \quad \forall \ell \geq 1,
$$

where $S, T$ are i.i.d. from $\nu$. This concept was introduced in Benjamini et al. (1998, Theorem 1.3), where it is shown that such a prior exists on infinite paths $(k=\infty)$ in the infinite $d$-dimensional integer lattice $(m=\infty)$ when $d=3$. In fact, it is constructed with support on oriented paths taking steps in $\{(1,0,0),(0,1,0),(0,0,1)\}$. Obviously, in the finite case, it suffices to restrict such a prior on the first $k-1$ steps and property (3.2) still holds when $k \leq m$, which we assume henceforth. ${ }^{1}$ Note that we can use the same prior when $d \geq 3$, by embedding the 3-dimensional integer lattice into the $d$-dimensional integer lattice. Because of this, we can take $\eta$ to be a numeric constant not depending on the dimension $d$.

Thus consider a prior $\nu$ satisfying (3.2). Then, for any $a>0$ small enough that $e^{a} \eta<1$,

$$
\begin{aligned}
\mathbb{E}_{\nu \otimes \nu}[\exp (a|S \cap T|)] & =\sum_{\ell \geq 1} e^{a \ell} \mathbb{P}_{\nu \otimes \nu}(|S \cap T|=\ell) \\
& =e^{a}+\sum_{\ell \geq 2}\left(e^{a \ell}-e^{a(\ell-1)}\right) \mathbb{P}_{\nu \otimes \nu}(|S \cap T| \geq \ell) \\
& \leq \Xi(a):=e^{a}+C_{0} \frac{\left(e^{a}-1\right) e^{a} \eta^{2}}{1-e^{a} \eta}
\end{aligned}
$$

We use this upper bound in Theorem 2.1 with $a=\lambda(|\psi|)$ and get that $\bar{R}_{\nu, \psi}^{*} \geq 1 / 2$ when $|\psi|$ is sufficiently small that $\eta \exp (\lambda(|\psi|))<1$ and $\Xi(\lambda(|\psi|)) \leq 2$. This is possible since $\lambda(|\psi|) \rightarrow 0$ as $|\psi| \rightarrow 0$ and $\Xi(a) \rightarrow 1$ as $a \rightarrow 0$.

\footnotetext{
${ }^{1}$ Clearly, an upper bound on $k$ is needed for there are no self-avoiding paths when $k>n$.
} 
Conversely, there exists a positive constant depending only on the dimension $d$ such that, if $\psi$ is larger than that constant, then the test defined in (2.2) asymptotically separates the hypotheses. This comes from a simple application of Proposition 2.3 together with the fact that, in the $d$-dimensional integer lattice, there are at most $(2 d)^{k-1}$ paths of length $k$ starting at a given node. Following Remark 2.4, we can handle the case when $|\psi|$ is large enough in a similar way. From this discussion, we arrive at the following.

Corollary 3.1. Consider the d-dimensional integer lattice (3.1) with $d \geq 3$ seen as a torus. Let $\mathcal{C}$ denote the class of self-avoiding paths of length $k \leq m$ starting at a known location. Assume the autocorrelation coefficient $\psi$ is fixed. There exist constants $0<C_{1} \leq C_{2}<1$ depending only on $d$ such that, when $|\psi|<C_{1}$, $\liminf _{k \rightarrow \infty} R_{\mathcal{C}, \psi}^{*}>0$, while when $|\psi|>C_{2}, \lim _{k \rightarrow \infty} R_{\mathcal{C}, \psi}^{*}=0$.

Remark 3.2. As long as $k \leq m$, the size of the lattice does not matter since the starting location is known. Therefore, an asymptotic analysis is necessarily in terms of large $k$.

Remark 3.3. We conjecture that the constants in Corollary 3.1 are identical, meaning, that $C_{1}=C_{2}$. Our arguments show that this is so if we replace liminf with limsup in the statement, for in that case one can take

$$
C_{1}=C_{2}=C_{\ddagger}:=\inf \left\{C>0: \lim _{k \rightarrow \infty} R_{\mathcal{C}, \psi}^{*}=0 \text { when }|\psi|>C\right\},
$$

and the corollary implies that $C_{\ddagger} \in(0,1)$.

3.2. Unknown starting point. Let $\mathcal{C}$ be the class of all self-avoiding paths with $k$ nodes in $\mathcal{V}$. Assume that $m=n^{1 / d}$ is a multiple of $2 k$ for simplicity. To define the prior, we partition the lattice into hypercubes of side length $2 k-1$, indexed by $J$, and let $v_{j}$ denote the center of the hypercube $j \in J$. The number of such hypercubes satisfies $|J| \sim(m / 2 k)^{d}=n /(2 k)^{d}$. Still in dimension $d \geq 3$, let $\nu_{j}$ be a prior on self-avoiding paths starting at $v_{j}$ satisfying (3.2) and let $\nu$ be the even mixture of all these priors. Noting that paths starting from different origin nodes cannot intersect, for any $a>0$ small enough that $e^{a} \eta<1$,

$$
\mathbb{E}_{\nu \otimes \nu}[\exp (a|S \cap T|)]=1-\frac{1}{|J|}+\frac{1}{|J|} \mathbb{E}_{\nu_{j} \otimes \nu_{j}}[\exp (a|S \cap T|)] \leq 1+\frac{\Xi(a)}{|J|},
$$

where $j \in J$ is arbitrary. We use this upper bound in Theorem 2.1 with $a=\lambda(|\psi|)$ and get that $\bar{R}_{\nu, \psi}^{*} \geq 1-\frac{1}{2} \sqrt{2 /|J|}$ when $|\psi|$ is so small that $\eta \exp (\lambda(|\psi|))<1$ and $\Xi(\lambda(|\psi|)) \leq 2$. Noting that $|J| \rightarrow \infty$ when $m \gg k$ (i.e., the size of the grid dominates the path length), we see that $\bar{R}_{\nu, \psi}^{*} \rightarrow 1$.

Conversely, assume that $k / \log n \geq C_{0}$ for some positive constant $C_{0}$. Then there exists another positive constant depending only on the dimension $d$ and $C_{0}$ such that, if $\psi$ is larger than that constant, the test defined in (2.2) asymptotically separates the hypotheses. This comes from Proposition 2.3 and the fact that, in the $d$-dimensional integer lattice (3.1) with a total of $n=m^{d}$ nodes, there are at most $n(2 d)^{k-1}$ paths of length $k$. Following Remark 2.4, we can handle the case where $|\psi|$ is large enough in a similar way. We thus arrive at the following.

Corollary 3.4. Consider the context of Corollary 3.1 but now assuming that the starting location is unknown and that $k / \log m \geq C_{0}$ for some constant $C_{0}>0$. 
There exist constants $0<C_{1} \leq C_{2}<1$ depending only on $d$ and $C_{0}$ such that, when $|\psi|<C_{1}, \lim _{m \rightarrow \infty} R_{\mathcal{C}, \psi}^{*}=1$, while when $|\psi|>C_{2}, \lim _{m \rightarrow \infty} R_{\mathcal{C}, \psi}^{*}=0$.

Remark 3.5. As in Remark 3.3, we conjecture that we may take $C_{1}=C_{2}$, defined as in (3.3). (This definition would lead to a different constant in the present setting.)

Remark 3.6. The constants in Corollary 3.1 and Corollary 3.4 are implicit. Our analysis provides some nontrivial bounds on these constants. However, it is not precise enough to lead to the exact values. We note that the same is true in the (simpler) detection-of-means setting of Arias-Castro et al. (2008). Also, we reveal a regime where the minimal correlation coefficient $\psi$ allowing asymptotic hypotheses separation (i.e., $R_{\mathcal{C}, \psi}^{*} \rightarrow 0$ ) is bounded away from 0 and 1 when both $k$ and $n$ go to infinity. (The situation is qualitatively different in dimension $d=2$ and we refer the reader to Arias-Castro et al., 2008 for a detailed treatment of that case in the detection-of-means setting.)

\section{Proofs}

4.1. Preliminaries. Let $\boldsymbol{\Gamma}(\psi)$ denote the covariance operator of an (infinite) autoregressive model of order 1 with parameter coefficient $\psi$. The operator $\boldsymbol{\Gamma}(\psi)$ is positive definite and invertible when $|\psi|<1$. Note that any $S \in \mathcal{C}$ is homomorphic to $\{1, \ldots,|S|\}$, and identifying the two, we have $\left(\boldsymbol{\Gamma}_{S}(\psi)\right)_{i, j}=\psi^{|i-j|}$, where $\boldsymbol{\Gamma}_{S}(\psi)$ is the principal submatrix of the covariance operator $\boldsymbol{\Gamma}(\psi)$ indexed by $S$.

Any autoregressive process $Y=\left(Y_{i}\right)_{i \in \mathbb{Z}}$ of order 1 with parameter $\psi$ can be represented as a Gaussian Markov random field (GMRF) on the line. We have the decomposition

$$
Y_{i}=\phi Y_{i-1}+\phi Y_{i+1}+\epsilon_{i},
$$

where $\epsilon_{i} \sim \mathcal{N}\left(0, \sigma_{\phi}^{2}\right)$ is independent of $\left(Y_{j}\right)_{j \neq i}$ and

$$
\phi:=\frac{\psi}{1+\psi^{2}}, \quad \sigma_{\phi}^{2}:=\frac{1-\psi^{2}}{1+\psi^{2}} .
$$

We start with some simple remarks relating autoregressive processes of order 1 to GMRFs. See Guyon (1995, Sect. 1.3) for more details. This representation of a stationary autoregressive process enables us to adapt some of the arguments developed in Arias-Castro et al. (2015b) for stationary GMRFs.

Lemma 4.1. Identify $S$ with $(1, \ldots,|S|)$ and consider any $\psi \in(-1,1)$. Then

$$
\left(\boldsymbol{\Gamma}_{S}^{-1}(\psi)\right)_{i, j}= \begin{cases}1 / \sigma_{\phi}^{2} & \text { if } i=j \text { and } i \in\{2, \ldots,|S|-1\}, \\ 1 /\left(1-\psi^{2}\right) & \text { if } i \in\{1,|S|\}, \\ -\phi / \sigma_{\phi}^{2} & \text { if }|i-j|=1, \\ 0 & \text { if }|i-j|>1 .\end{cases}
$$

Proof: We leave $\psi$ implicit and write $\boldsymbol{\Gamma}$ for $\boldsymbol{\Gamma}(\psi)$. Denote by $Y_{S}$ the restriction of the stationary autoregressive process $Y$ to $S$. First consider any index $i \in\{2, \ldots,|S|-$ 1\}. By the Markov property (4.1), conditionally to $\left(Y_{i-1}, Y_{i+1}\right), Y_{i}$ is independent to all the remaining variables. Thus, the conditional distribution of $Y_{i}$ given $\left(Y_{j}\right)_{j \neq i}$ is the same as the conditional distribution of $Y_{i}$ given $\left(Y_{j}\right)_{j \in S \backslash\{i\}}$. This conditional distribution characterizes the $i$-th row of the inverse covariance matrix $\boldsymbol{\Gamma}_{S}^{-1}$. More precisely, the conditional variance $\sigma_{\phi}^{2}$ of $Y_{i}$ given $Y_{S}$ is $\left[\left(\boldsymbol{\Gamma}_{S}^{-1}\right)_{i, i}\right]^{-1}$. Furthermore, $-\left(\boldsymbol{\Gamma}^{-1}\right)_{i, j} /\left(\boldsymbol{\Gamma}^{-1}\right)_{i, i}$ is the $j$-th parameter of the conditional regression (4.1) of $Y_{i}$ 
given $\left(Y_{j}\right)_{j \neq i}$, and therefore we conclude that $\left(\boldsymbol{\Gamma}^{-1}\right)_{i, i}=\left(\sigma_{\phi}^{2}\right)^{-1}=\left(\boldsymbol{\Gamma}_{S}^{-1}\right)_{i, i}$ and $\left(\boldsymbol{\Gamma}^{-1}\right)_{i, j} /\left(\boldsymbol{\Gamma}^{-1}\right)_{i, i}$ equals $-\phi$ if $|j-i|=1$ and is zero otherwise.

Now consider the case $i=|S|, i=1$ being handled similarly. Since $\boldsymbol{\Gamma}_{S}^{-1}$ is a symmetric matrix, we only have to compute $\left(\boldsymbol{\Gamma}_{S}^{-1}\right)_{|S|,|S|}$ and $\left(\boldsymbol{\Gamma}_{S}^{-1}\right)_{|S|, 1}$. By definition of autoregressive processes, we have

$$
Y_{|S|}=\psi Y_{|S|-1}+\omega_{|S|},
$$

where $\omega_{|S|} \sim \mathcal{N}\left(0,1-\psi^{2}\right)$ is independent of $\left(Y_{1}, \ldots Y_{|S|-2}\right)$. The above expression characterizes the conditional regression of $Y_{|S|}$ given $\left(Y_{1}, \ldots Y_{|S|-1}\right)$. Arguing as previously, we conclude that $\left(\boldsymbol{\Gamma}^{-1}\right)_{|S|, 1}=0$ and $\left(\boldsymbol{\Gamma}^{-1}\right)_{|S|,|S|}=1 /\left(1-\psi^{2}\right)$.

We let $\|\mathbf{A}\|$ denote the operator norm of a matrix $\mathbf{A}$.

Lemma 4.2. Let $\mathbf{A}$ and $\mathbf{B}$ be (complex or real) matrices of same dimensions. Let $\operatorname{col}(\mathbf{A})$ index the column vectors of $\mathbf{A}$ that are nonzero. Then

$$
\left|\operatorname{Tr}\left(\mathbf{A}^{\top} \mathbf{B}\right)\right| \leq|\operatorname{col}(\mathbf{A}) \cap \operatorname{col}(\mathbf{B})|\|\mathbf{A}\|\|\mathbf{B}\| .
$$

Proof: Define the index set $J=\operatorname{col}(\mathbf{A}) \cap \operatorname{col}(\mathbf{B})$. Let $\mathbf{A}_{J}$ denote the submatrix of A with columns indexed by $J$, and define $\mathbf{B}_{J}$ similarly. We then have

$$
\left|\operatorname{Tr}\left(\mathbf{A}^{\top} \mathbf{B}\right)\right|=\left|\operatorname{Tr}\left(\mathbf{A}_{J}^{\top} \mathbf{B}_{J}\right)\right| \leq|J|\left\|\mathbf{A}_{J}^{\top} \mathbf{B}_{J}\right\| \leq|J|\left\|\mathbf{A}_{J}\right\|\left\|\mathbf{B}_{J}\right\| \leq|J|\|\mathbf{A}\|\|\mathbf{B}\| .
$$

4.2. Proof of Theorem 2.1. Recall that with $\psi$ fixed, $\boldsymbol{\Gamma}_{S}$ denotes the covariance matrix of an autoregressive model of order 1 of length $|S|$ and with parameter $\psi$. As is well-known (and explained in Arias-Castro et al., 2015b for example),

$$
\bar{R}_{\nu}^{*}=1-\frac{1}{2} \mathbb{E}_{0}\left|L_{\nu}(X)-1\right| \geq 1-\frac{1}{2} \sqrt{\mathbb{E}_{0}\left[L_{\nu}(X)^{2}\right]-1},
$$

where

$$
L_{\nu}(x):=\sum_{S \in \mathcal{C}} \nu(S) L_{S}(x)
$$

with

$$
L_{S}(x):=\exp \left(\frac{1}{2} x_{S}^{\top}\left(\mathbf{I}_{S}-\boldsymbol{\Gamma}_{S}^{-1}\right) x_{S}-\frac{1}{2} \log \operatorname{det}\left(\boldsymbol{\Gamma}_{S}\right)\right) .
$$

We thus need to upper bound

$$
\mathbb{E}_{0}\left[L_{\nu}(X)^{2}\right]=\sum_{S, T \in \mathcal{C}} \nu(S) \nu(T) \mathbb{E}_{0}\left[L_{S}(X) L_{T}(X)\right] .
$$

Unlike in the setting that concerns Arias-Castro et al. (2015b), here two subgraphs $S$ and $T$ in the support of the prior $\nu$ may not be disjoint, and if $S \cap T \neq \emptyset, L_{S}(X)$ and $L_{T}(X)$ are not independent.

Before we proceed, we leave the dependency in $X$ implicit and we formally index the elements of $S$ by $\{1, \ldots, k\}$. By Lemma 4.1, we have

$$
1 \leq\left(\boldsymbol{\Gamma}_{S}^{-1}\right)_{1,1}=\left(\boldsymbol{\Gamma}_{S}^{-1}\right)_{k, k}=\frac{1}{1-\psi^{2}} \leq\left(\boldsymbol{\Gamma}_{S}^{-1}\right)_{i, i}=\frac{1}{\sigma_{\phi}^{2}}, \quad \forall i \in\{2, \ldots k-1\},
$$

and

$$
\left(\boldsymbol{\Gamma}_{S}^{-1}\right)_{i, i+1}=\left(\boldsymbol{\Gamma}_{S}^{-1}\right)_{i, i-1}=-\frac{\phi}{\sigma_{\phi}^{2}}
$$


while all the other entries of $\boldsymbol{\Gamma}_{S}^{-1}$ are zero. Hence, the matrix $\mathbf{A}_{S}:=\mathbf{I}_{S}-\boldsymbol{\Gamma}_{S}^{-1}$ satisfies

$$
\left\|\mathbf{A}_{S}\right\| \leq \sup _{j \in S} \sum_{i \in S}\left|\left(\mathbf{A}_{S}\right)_{i, j}\right| \leq \frac{2|\phi|}{\sigma_{\phi}^{2}}+\frac{1-\sigma_{\phi}^{2}}{\sigma_{\phi}^{2}}=\frac{2|\psi|}{1-|\psi|},
$$

where we used the expressions (4.2) of $\phi$ and $\sigma_{\phi}$. Let us fix $S$ and $T$ two anomalous subsets in $\mathcal{C}$. In the following $\widetilde{\boldsymbol{\Gamma}}_{S}$ (resp. $\widetilde{\boldsymbol{\Gamma}}_{T}$ ) denotes the covariance of $X_{S \cup T}$ when $X \sim \mathbb{P}_{S, \psi}$ (resp. $X \sim \mathbb{P}_{T, \psi}$ ). Note that the restriction of $\widetilde{\boldsymbol{\Gamma}}_{S}$ to $S \times S$ is exactly $\boldsymbol{\Gamma}_{S}$ whereas its restriction to $(T \backslash S) \times(T \backslash S)$ is the identity matrix. We have

$$
\begin{aligned}
\mathbb{E}\left[L_{S} L_{T}\right]=\mathbb{E}\left[\operatorname { e x p } \left(X_{S \cup T}^{\top}\left(\mathbf{I}_{S \cup T}-\frac{1}{2} \widetilde{\boldsymbol{\Gamma}}_{S}^{-1}-\frac{1}{2} \widetilde{\boldsymbol{\Gamma}}_{T}^{-1}\right) X_{S \cup T}\right.\right. & \left.\left.\quad-\frac{1}{2} \log \operatorname{det}\left(\boldsymbol{\Gamma}_{S}\right)-\frac{1}{2} \log \operatorname{det}\left(\boldsymbol{\Gamma}_{T}\right)\right)\right] \\
= & \exp \left(-\frac{1}{2} \log \operatorname{det}\left(\widetilde{\boldsymbol{\Gamma}}_{S}^{-1}+\widetilde{\boldsymbol{\Gamma}}_{T}^{-1}-\mathbf{I}_{S \cup T}\right)-\frac{1}{2} \log \operatorname{det}\left(\boldsymbol{\Gamma}_{S}\right)-\frac{1}{2} \log \operatorname{det}\left(\boldsymbol{\Gamma}_{T}\right)\right) .
\end{aligned}
$$

We used the fact that

$$
\left\|\mathbf{I}_{S \cup T}-\frac{1}{2} \widetilde{\boldsymbol{\Gamma}}_{S}^{-1}-\frac{1}{2} \widetilde{\boldsymbol{\Gamma}}_{T}^{-1}\right\| \leq \frac{1}{2}\left\|\mathbf{A}_{S}\right\|+\frac{1}{2}\left\|\mathbf{A}_{T}\right\| \leq \frac{2|\psi|}{1-|\psi|}<\frac{1}{2},
$$

by (4.6) and the fact that $|\psi| \leq 1 / 5$. Define $\widetilde{\mathbf{A}}_{S}=\mathbf{I}_{S \cup T}-\widetilde{\boldsymbol{\Gamma}}_{S}^{-1}$ and $\widetilde{\mathbf{A}}_{T}$ similarly. Using these bounds, together with the fact that, for a symmetric matrix $\mathbf{B}$ with operator norm strictly less than 1 ,

$$
\log \operatorname{det}(\mathbf{I}-\mathbf{B})=\operatorname{Tr} \log (\mathbf{I}-\mathbf{B})=-\sum_{\ell=1}^{\infty} \frac{1}{\ell} \operatorname{Tr}\left(\mathbf{B}^{\ell}\right),
$$

we get

$$
\begin{aligned}
\Lambda:= & -\frac{1}{2} \log \operatorname{det}\left(\widetilde{\boldsymbol{\Gamma}}_{S}^{-1}+\widetilde{\boldsymbol{\Gamma}}_{T}^{-1}-\mathbf{I}_{S \cup T}\right)-\frac{1}{2} \log \operatorname{det}\left(\boldsymbol{\Gamma}_{S}\right)-\frac{1}{2} \log \operatorname{det}\left(\boldsymbol{\Gamma}_{T}\right) \\
= & -\frac{1}{2} \log \operatorname{det}\left(\mathbf{I}_{S \cup T}-\widetilde{\mathbf{A}}_{S}-\widetilde{\mathbf{A}}_{T}\right) \\
& \quad+\frac{1}{2} \log \operatorname{det}\left(\mathbf{I}_{S \cup T}-\widetilde{\mathbf{A}}_{S}\right)+\frac{1}{2} \log \operatorname{det}\left(\mathbf{I}_{S \cup T}-\widetilde{\mathbf{A}}_{T}\right) \\
= & \frac{1}{2} \sum_{\ell=1}^{\infty} \frac{1}{\ell}\left(\operatorname{Tr}\left[\left(\widetilde{\mathbf{A}}_{S}+\widetilde{\mathbf{A}}_{T}\right)^{\ell}\right]-\operatorname{Tr}\left[\widetilde{\mathbf{A}}_{S}^{\ell}\right]-\operatorname{Tr}\left[\widetilde{\mathbf{A}}_{T}^{\ell}\right]\right) \\
= & \frac{1}{2} \sum_{\ell=2}^{\infty} \frac{1}{\ell} \sum_{(s, t) \in Q_{\ell}} \operatorname{Tr}\left[\widetilde{\mathbf{A}}_{S}^{s_{1}} \widetilde{\mathbf{A}}_{T}^{t_{1}} \cdots \widetilde{\mathbf{A}}_{S}^{s_{\ell}} \widetilde{\mathbf{A}}_{T}^{t_{\ell}}\right],
\end{aligned}
$$

where

$$
Q_{\ell}:=\left\{(s, t) \in\left(\{0,1\}^{\ell} \backslash\{0\}^{\ell}\right)^{2}: s_{1}+\cdots+s_{\ell}+t_{1}+\cdots+t_{\ell}=\ell\right\} .
$$

For any $(s, t) \in Q_{\ell}$, there exists $j$ such that either $s_{j}=t_{j}=1$ or $t_{j-1}=s_{j}=1$. For example, assuming the former holds, we apply Lemma 4.2 to get

$$
\begin{aligned}
\operatorname{Tr}\left[\widetilde{\mathbf{A}}_{S}^{s_{1}} \widetilde{\mathbf{A}}_{T}^{t_{1}} \cdots \widetilde{\mathbf{A}}_{S}^{s_{\ell}} \widetilde{\mathbf{A}}_{T}^{t_{\ell}}\right] & =\operatorname{Tr}\left[\left(\widetilde{\mathbf{A}}_{S}^{s_{1}} \widetilde{\mathbf{A}}_{T}^{t_{1}} \cdots \widetilde{\mathbf{A}}_{S}^{s_{j}}\right)\left(\widetilde{\mathbf{A}}_{T}^{t_{j}} \cdots \widetilde{\mathbf{A}}_{S}^{s_{\ell}} \widetilde{\mathbf{A}}_{T}^{t_{\ell}}\right)\right] \\
& \leq|S \cap T|\left\|\widetilde{\mathbf{A}}_{S}^{s_{1}} \widetilde{\mathbf{A}}_{T}^{t_{1}} \cdots \widetilde{\mathbf{A}}_{S}^{s_{j}}\right\|\left\|\widetilde{\mathbf{A}}_{T}^{t_{j}} \cdots \widetilde{\mathbf{A}}_{S}^{s_{\ell}} \widetilde{\mathbf{A}}_{T}^{t_{\ell}}\right\| \\
& \leq|S \cap T|\left\|\widetilde{\mathbf{A}}_{S}\right\|^{s_{1}}\left\|\widetilde{\mathbf{A}}_{T}\right\|^{t_{1}} \cdots\left\|\widetilde{\mathbf{A}}_{S}\right\|^{s_{\ell}}\left\|\widetilde{\mathbf{A}}_{T}\right\|^{t_{\ell}} \\
& \leq|S \cap T| \zeta^{\ell}, \quad \text { where } \zeta=\frac{2|\psi|}{1-|\psi|} .
\end{aligned}
$$


Note that the last line comes from (4.7).

With this, together with the fact that $\left|Q_{\ell}\right| \leq\left(\begin{array}{c}2 \ell \\ \ell\end{array}\right)$ and the identity $(1-x)^{-1 / 2}=$ $\sum_{n \geq 0}\left(\begin{array}{c}2 n \\ n\end{array}\right)(x / 4)^{n}$ for $x \in(0,1)$, and noting that $\zeta<1 / 4$ when $|\psi|<1 / 9$, we obtain

$$
\Lambda \leq \frac{1}{2}|S \cap T| \sum_{\ell \geq 2} \frac{1}{\ell}\left(\begin{array}{c}
2 \ell \\
\ell
\end{array}\right) \zeta^{\ell} \leq \frac{1}{4}|S \cap T|\left[(1-4 \zeta)^{-1 / 2}-1-2 \zeta\right] .
$$

We then conclude with (4.5) and the expression of $\zeta$ in terms of $\psi$.

4.3. Proof of Proposition 2.3. All through, we leave the dependence of $t$ in $k$ implicit.

Under the null. We first control $V_{t}^{*}$ under the null hypothesis. For simplicity, assume that $k$ is even and decompose the statistics $V_{t, S}$ into $V_{t, S}=V_{1, t, S}+V_{2, t, S}$, where

$$
V_{1, t, S}:=\sum_{j=1}^{k / 2} V_{t, S}(2 j), \quad \text { and } \quad V_{2, t, S}:=\sum_{j=2}^{k / 2} V_{t, S}(2 j-1),
$$

so that all the terms in $V_{1, t, S}$ (resp. $V_{2, t, S}$ ) are independent. Set $V_{1, t}^{*}=\max _{S \in \mathcal{C}} V_{1, t, S}$ and $V_{2, t}^{*}=\max _{S \in \mathcal{C}} V_{2, t, S}$. By symmetry, it suffices to bound $V_{1, t}^{*}$.

For any $S \in \mathcal{C}$, we have $V_{1, t, S} \sim \operatorname{Bin}\left(k / 2, p_{t}\right)$. Thus, for any $S \in \mathcal{C}$, with the union bound, we have

$$
\mathbb{P}_{0}\left\{V_{1, t}^{*} \geq v\right\} \leq|\mathcal{C}| \mathbb{P}_{0}\left\{V_{1, t, S} \geq v\right\} \leq|\mathcal{C}| \mathbb{P}\left\{\operatorname{Bin}\left(k / 2, p_{t}\right) \geq v\right\} .
$$

Define $b_{t}=1 /\left(2 p_{t}\right)$ and $\varphi(b)=b(\log b-1)+1$. Choosing $v=k / 4=b_{t} k / 2 p_{t}$, and using Bennett's inequality, the right-hand side is bounded by

$$
\begin{aligned}
|\mathcal{C}| \exp \left(-k / 2 p_{t} \varphi\left(b_{t}\right)\right) & =\exp \left(\log |\mathcal{C}|-(k / 2) p_{t} \varphi\left(b_{t}\right)\right) \\
& =\exp \left(\log |\mathcal{C}|-(k / 2) \frac{1}{2} h\left(2 p_{t}\right)\right) \\
& \leq \exp \left(\left(k / 8-(k / 2) \frac{1}{2}\right) h\left(2 p_{t}\right)\right) \\
& \leq \exp (-k / 8),
\end{aligned}
$$

where the inequality holds eventually as the sample size increases.

Thus we found that

$$
\begin{aligned}
\mathbb{P}_{0}\left\{V_{t}^{*} \geq k / 2\right\} & \leq \mathbb{P}_{0}\left\{V_{1, t}^{*} \geq k / 4\right\}+\mathbb{P}_{0}\left\{V_{2, t}^{*} \geq k / 4\right\} \\
& \leq \exp (-k / 8)+\exp (-k / 8)=2 \exp (-k / 8) .
\end{aligned}
$$

Under the alternative. We now consider the alternative hypothesis. Let $(S, \psi) \in$ $\mathcal{C} \times(-1,1)$ denote the anomalous path and the autoregressive parameter. Recall that we assume that $\psi \geq 1-\left(t / \mathrm{N}^{-1}(4 / 5)\right)^{2}$. Denote $S=\left(s_{1}, \ldots, s_{k}\right)$. By definition, $V_{t}^{*} \geq V_{t, S}$. Define $Z_{j}:=\left(X_{s_{j+1}}-X_{s_{j}}\right) / \sqrt{2(1-\psi)}$ for any $j \in\{1, \ldots, k-1\}$. We have $Z_{j} \sim \mathcal{N}(0,1)$ and $\mathbb{E}\left[Z_{j} Z_{j^{\prime}}\right]=\psi^{\left|j-j^{\prime}\right|-1}(\psi-1) / 2$ for $j \neq j^{\prime}$. Define $q=2 \mathrm{~N}(t / \sqrt{1-\psi})-1$, and note that $q \geq 3 / 5$ by our assumption on $\psi$.

First, in terms of expectation, we obtain the bound

$$
\mathbb{E}_{S}\left[V_{t, S}\right]=(k-1) q \geq(k-1) \frac{3}{5} .
$$

We now bound the variance. Fix any $j \neq j^{\prime}$, and denoting $a=\mathbb{E}\left[Z_{j} Z_{j^{\prime}}\right]$, define $U=\left(Z_{j}-a Z_{j^{\prime}}\right) / \sqrt{1-a^{2}}$. Note that $U \sim \mathcal{N}(0,1)$ is independent of $Z_{j^{\prime}}$. For any 
$x \in \mathbb{R}$, we have

$$
\begin{aligned}
\mathbb{P}\{\mid & \left.Z_{j}\left|\leq \frac{t}{\sqrt{1-\psi}}\right| Z_{j^{\prime}}=x\right\} \\
& =\mathbb{P}\left\{\frac{-t-a x}{\sqrt{1-\psi} \sqrt{1-a^{2}}} \leq U \leq \frac{t-a x}{\sqrt{1-\psi} \sqrt{1-a^{2}}}\right\} \\
& \leq \mathbb{P}\left\{|U| \leq \frac{t}{\sqrt{1-\psi} \sqrt{1-a^{2}}}\right\} \\
& =2 \mathbb{P}\left\{U \leq \frac{t}{\sqrt{1-\psi}}\right\}-1+2 \mathbb{P}\left\{\frac{t}{\sqrt{1-\psi}} \leq U \leq \frac{t}{\sqrt{1-\psi} \sqrt{1-a^{2}}}\right\} \\
& =q+2\left[\mathrm{~N}\left(\frac{t}{\sqrt{1-\psi} \sqrt{1-a^{2}}}\right)-\mathrm{N}\left(\frac{t}{\sqrt{1-\psi}}\right)\right] \\
& \leq q+2 \frac{t}{\sqrt{1-\psi}}\left[\frac{1}{\sqrt{1-a^{2}}}-1\right] \frac{1}{\sqrt{2 \pi}} \exp \left[-\frac{t^{2}}{2(1-\psi)}\right] \\
& \leq q+\left(\frac{4}{3}\right)^{3 / 2} a^{2} \frac{t}{\sqrt{1-\psi}} \frac{1}{\sqrt{2 \pi}} \exp \left[-\frac{t^{2}}{2(1-\psi)}\right] \\
& \leq q+a^{2} .
\end{aligned}
$$

We used the fact that $U$ is standard normal in the second and fourth line, a Taylor development (of order 1) in the fifth line, the fact that $|a| \leq 1 / 2$ in the sixth line, and the fact that $y e^{-y^{2}} \leq(2 e)^{-1 / 2}$ for any $y \geq 0$ in the last line. As a consequence,

$$
\begin{aligned}
\operatorname{Cov} & \left(\mathbb{I}\left\{\left|Z_{j}\right| \leq \frac{t}{\sqrt{1-\psi}}\right\}, \mathbb{I}\left\{\left|Z_{j^{\prime}}\right| \leq \frac{t}{\sqrt{1-\psi}}\right\}\right) \\
& =\int_{-\frac{t}{\sqrt{1-\psi}}}^{\frac{t}{\sqrt{1-\psi}}} \mathbb{P}\left\{\left|Z_{j}\right| \leq \frac{t}{\sqrt{1-\psi}} \mid Z_{j^{\prime}}=x\right\} \frac{e^{-x^{2} / 2}}{\sqrt{2 \pi}} \mathrm{d} x-q^{2} \\
& \leq\left(q+a^{2}\right) q-q^{2}=q a^{2}=q \psi^{2\left|j-j^{\prime}\right|-2}(1-\psi)^{2},
\end{aligned}
$$

for all $j \neq j^{\prime}$. We conclude that

$$
\begin{aligned}
\operatorname{Var}_{S}\left(V_{t, S}\right) & =\operatorname{Var}_{S}\left(\sum_{j=1}^{k-1} \mathbb{I}\left\{\left|Z_{j}\right| \leq \frac{t}{\sqrt{1-\psi}}\right\}\right) \\
& \leq(k-1)\left[q(1-q)+2 q(1-\psi)^{2} \sum_{i=1}^{\infty} \psi^{2(j-1)}\right] \\
& \leq(k-1)\left[q(1-q)+2 q \frac{1-\psi}{1+\psi}\right] \\
& \leq 3 \mathbb{E}_{S}\left[V_{t, S}\right] .
\end{aligned}
$$

Now, by Chebyshev's inequality, we have for $b>0$,

$$
\mathbb{P}_{S}\left\{V_{t, S} \geq \mathbb{E}_{S}\left[V_{t, S}\right]-b \sqrt{\operatorname{Var}_{S}\left[V_{t, S}\right]}\right\} \geq 1-1 / b^{2},
$$

with

$$
\mathbb{E}_{S}\left[V_{t, S}\right]-b \sqrt{\operatorname{Var}_{S}\left[V_{t, S}\right]} \geq \mathbb{E}_{S}\left[V_{t, S}\right]-b \sqrt{3 \mathbb{E}_{S}\left[V_{t, S}\right]},
$$


and choosing $b=\log k$, for $k$ large enough we have, in view of (4.8),

$$
\mathbb{E}_{S}\left[V_{t, S}\right]-b \sqrt{3 \mathbb{E}_{S}\left[V_{t, S}\right]} \geq k / 2 .
$$

When this is the case, we have

$$
\mathbb{P}_{S, \psi}\left\{V_{t}^{*}<k / 2\right\} \leq \mathbb{P}_{S, \psi}\left\{V_{t, S}<k / 2\right\} \leq 1 / b^{2}=1 /(\log k)^{2} .
$$

Risk. Thus, for $k$ large enough, say $k>k_{0}$ (where $k_{0}$ is a numerical constant), the risk of $f_{t}$ is bounded by $2 e^{-k / 8}+1 /(\log k)^{2}$. We conclude that the stated result holds with $r_{k}=2$ for $k \leq k_{0}$ and $r_{k}=2 e^{-k / 8}+1 /(\log k)^{2}$ for $k>k_{0}$.

\section{References}

L. Addario-Berry, N. Broutin, L. Devroye and G. Lugosi. On combinatorial testing problems. Ann. Statist. 38 (5), 3063-3092 (2010). MR2722464.

E. Arias-Castro, S. Bubeck and G. Lugosi. Detection of correlations. Ann. Statist. 40 (1), 412-435 (2012). MR3014312.

E. Arias-Castro, S. Bubeck and G. Lugosi. Detecting positive correlations in a multivariate sample. Bernoulli 21 (1), 209-241 (2015a). MR3322317.

E. Arias-Castro, S. Bubeck, G. Lugosi and N. Verzelen. Detecting markov random fields hidden in white noise. ArXiv Mathematics e-prints (2015b). arXiv: 1504.06984.

E. Arias-Castro, E. J. Candès and A. Durand. Detection of an anomalous cluster in a network. Ann. Statist. 39 (1), 278-304 (2011). MR2797847.

E. Arias-Castro, E. J. Candès, H. Helgason and O. Zeitouni. Searching for a trail of evidence in a maze. Ann. Statist. 36 (4), 1726-1757 (2008). MR2435454.

E. Arias-Castro, D. L. Donoho and X. Huo. Near-optimal detection of geometric objects by fast multiscale methods. IEEE Trans. Inform. Theory 51 (7), 24022425 (2005). MR2246369.

E. Arias-Castro, D. L. Donoho and X. Huo. Adaptive multiscale detection of filamentary structures in a background of uniform random points. Ann. Statist. 34 (1), 326-349 (2006). MR2275244.

E. Arias-Castro and G. R. Grimmett. Cluster detection in networks using percolation. Bernoulli 19 (2), 676-719 (2013). MR3037169.

I. Benjamini, R. Pemantle and Y. Peres. Unpredictable paths and percolation. Ann. Probab. 26 (3), 1198-1211 (1998). MR1634419.

B. DasGupta, J. P. Hespanha, J. Riehl and E. Sontag. Honey-pot constrained searching with local sensory information. Nonlinear Anal. 65 (9), 1773-1793 (2006). MR2252129.

A. Desolneux, L. Moisan and J.-M. Morel. Maximal meaningful events and applications to image analysis. Ann. Statist. 31 (6), 1822-1851 (2003). MR2036391.

X. Guyon. Random fields on a network. Probability and its Applications (New York). Springer-Verlag, New York (1995). ISBN 0-387-94428-1. MR1344683.

B. Mukherjee, L.T. Heberlein and K.N. Levitt. Network intrusion detection. IEEE Network 8 (3), 26-41 (1994). DOI: 10.1109/65.283931.

G. Walther. Optimal and fast detection of spatial clusters with scan statistics. Ann. Statist. 38 (2), 1010-1033 (2010). MR2604703.

Y. Zhang and W. Lee. Intrusion detection in wireless ad-hoc networks. In International Conference on Mobile Computing and Networking (MobiCom), pages 275-283. ACM (2000). 